\title{
Percolation approach to glassy dynamics with continuously broken ergodicity
}

\author{
Jeferson J. Arenzon, ${ }^{1, *}$ Antonio Coniglio, ${ }^{2,3}$ Annalisa Fierro, ${ }^{2,3}$ and Mauro Sellitto ${ }^{4}$ \\ ${ }^{1}$ Instituto de Física, Universidade Federal do Rio Grande do Sul, CP 15051, 91501-970 Porto Alegre RS, Brazil \\ ${ }^{2}$ Dipartimento di Scienze Fisiche, Università di Napoli "Federico II," Complesso Universitario di Monte Sant'Angelo, \\ Via Cintia, I-80126 Napoli, Italy \\ ${ }^{3}$ CNR-SPIN, Via Cintia, I-80126 Napoli, Italy \\ ${ }^{4}$ Dipartimento di Ingegneria Industriale e dell'Informazione, Seconda Università di Napoli, Real Casa dell'Annunziata, \\ I-81031 Aversa (CE), Italy
}

(Received 28 February 2014; revised manuscript received 27 June 2014; published 26 August 2014)

\begin{abstract}
We show that the relaxation dynamics near a glass transition with continuous ergodicity breaking can be endowed with a geometric interpretation based on percolation theory. At the mean-field level this approach is consistent with the mode-coupling theory (MCT) of type-A liquid-glass transitions and allows one to disentangle the universal and nonuniversal contributions to MCT relaxation exponents. Scaling predictions for the time correlation function are successfully tested in the $F_{12}$ schematic model and facilitated spin systems on a Bethe lattice. Our approach immediately suggests the extension of MCT scaling laws to finite spatial dimensions and yields predictions for dynamic relaxation exponents below an upper critical dimension of 6 .
\end{abstract}

DOI: 10.1103/PhysRevE.90.020301

PACS number(s): 64.70.qj, 64.60.ah, 64.70.P-

Percolation [1] is one of the most appealing examples of phase transitions which has been successfully applied to a variety of problems and has provided a deeper insight into the theory of critical phenomena. Although its relevance to amorphous magnets and structural glasses has been often suggested, the geometrical interpretation of scaling laws observed during glassy relaxation has been thwarted by several difficulties and remains one of the fundamental issues of condensed matter science.

In this Rapid Communication, we formulate a percolation approach to glassy dynamics with continuously broken ergodicity and exploit its predictions to provide an interpretation of the mode-coupling theory (MCT) of a continuous (or type-A) liquid-glass transition [2] and to suggest some scaling relations. Two key questions lie at the heart of our work: Is there any universality - in the sense of critical phenomenahidden in MCT exponents? Is there any geometrical picture underlying MCT scaling laws?

We develop our formalism for a generic spatial dimension and in the mean-field limit, but our approach can in principle be applied to a range of different systems whenever clusters play an essential role. The mean-field limit will make transparent and explicit the connection with MCT and will naturally yield a finite-dimensional extension of MCT scaling laws. Specifically, we generalize to finite dimensions the universal scaling law relating the exponents of structural relaxation time and critical decay law, and uncover an intermediate relaxation regime close to criticality. In this regime, the time correlation function is a combination of algebraic and stretched exponential decay, with precise predictions in terms of percolation critical exponents, and universal scaling relations. Comparisons with the analytical and numerical solution of the schematic MCT equation, and with Monte Carlo simulation of facilitated spin models on a Bethe lattice, give excellent results.

*arenzon@if.ufrgs.br
Schematic mode-coupling theory. We first recall the MCT results for the type-A liquid-glass transition. We focus on the schematic $F_{12}$ model which is known to reproduce the basic features of glassy dynamics [2]. In this case, the memory kernel functional $\mathrm{M}$ is

$$
\mathrm{M}[\phi(t)]=v_{1} \phi(t)+v_{2} \phi^{2}(t)
$$

where $v_{1}, v_{2}$ are parameters controlling the system state and the correlator $\phi(t)$ of density fluctuations at time $t$ obeys the integrodifferential equation

$$
\phi(t)+t_{0} \dot{\phi}(t)+\int_{0}^{t} \mathrm{M}[\phi(t-s)] \dot{\phi}(s) d s=0,
$$

where $t_{0}$ is a characteristic microscopic time scale and overdamped local motion is assumed for simplicity. We are interested here in the region of the phase diagram in which the ergodic-nonergodic transition is continuous. Continuous glass transitions have recently attracted significant attention in connection with the behavior of fluids confined in porous media [3-5], which is related to the diffusion-localization behavior of the Lorentz gas. In this context the relevance of percolation was previously noticed [6].

In the $F_{12}$ schematic model the continuous transition line corresponds to the segment $v_{1}=1$ and $v_{2} \in[0,1]$. At criticality relaxation is algebraic, $\phi(t) \sim t^{-a}$, and the structural relaxation time $\tau_{\epsilon}$ at distance $\epsilon$ from the critical line behaves as $\tau_{\epsilon} \sim|\epsilon|^{-\zeta}$ near the transition. The exponents $a$ and $\zeta$ are not independent but connected throughout the continuous glass transition range by [2]

$$
\lambda=\frac{1}{2} \frac{\mathrm{M}^{\prime \prime}(q)}{\left[\mathrm{M}^{\prime}(q)\right]^{3 / 2}}, \quad \zeta=\frac{1}{a}, \quad \frac{\Gamma^{2}(1-a)}{\Gamma(1-2 a)}=\lambda,
$$

where $\Gamma$ is the Euler's gamma function, the kernel derivatives relative to $\phi$ are computed at criticality, and $\lambda$ is the so-called exponent parameter. For the $\mathrm{F}_{12}$ schematic model $\lambda=v_{2} / v_{1}^{3 / 2}$ and, in particular, $\lambda=v_{2}$ in the continuous glass transition range. In the frozen phase the Edwards-Anderson order 
parameter $q=\lim _{t \rightarrow \infty} \phi(t)$ approaches zero as $q \sim \epsilon^{\beta}$ with $\beta=1$, which coincides with the mean-field value of the order parameter percolation exponent. Following this hint we develop a dynamical percolation model describing relaxation dynamics near the type-A transition.

Before we proceed, the rather peculiar nature of MCT should be emphasized: On one hand, it gives rise to relaxation patterns with a universal scaling relation between the exponents $\zeta$ and $a$; on the other hand, these exponents must be considered nonuniversal, because they depend on the kernel parameters. Further, the MCT glass transition has a purely dynamical nature unrelated to any equilibrium singularity. These unconventional features are a major stumbling block in understanding the glassy relaxation as an ordinary dynamical critical phenomenon (see, however, Refs. [7-11] for recent progress in this direction). In our cluster interpretation of MCT we connect the parameter $\lambda$ to the cluster lifetime and disentangle the universal and nonuniversal features encoded in the exponents $a, \zeta$, and $\lambda$. These features turn out to be related to the underlying geometric structure of percolation clusters and to the cluster lifetime. From this connection, universal scaling relations valid in generic spatial dimensions will follow.

Percolation approach. We first note that the MCT relation $\zeta=1 / a$ can be generally understood in terms of a simple scaling argument. Indeed, if we write

$$
\phi(t, \epsilon)=\epsilon^{\beta} \mathcal{F}\left(\frac{t}{\tau_{\epsilon}}\right),
$$

where the scaling function $\mathcal{F}(u)$ has limiting behaviors,

$$
\mathcal{F}(u) \propto \begin{cases}u^{-\beta / \zeta}, & \text { for } \quad u \ll 1, \\ \text { const, } & \text { for } \quad u \gg 1,\end{cases}
$$

then relation $\zeta=1 / a$ is correctly recovered with $\beta=1$.

To explicitly obtain the scaling function we introduce a percolation approach elaborating on the cluster formulation used to describe the sol-gel transition [12]. We posit that our reference glassy system can be described as a collection of clusters, each of which decays exponentially in time over a time scale $t_{s}$ that increases with the cluster size $s$. The structure and relaxation of clusters evidently depend on the precise nature of the system interaction and the underlying microscopic dynamics. We shall assume a power-law behavior of a cluster lifetime, $t_{s} \propto s^{x}$, as borne out from experimental results and simulations on polymers [13]. Under these hypotheses the correlator $\phi(t)$, describing global relaxation, can be written as the superposition of the decay of different clusters:

$$
\phi(t)=\sum_{s} s n(s) \exp \left(-\frac{t}{t_{s}}\right) / \sum_{s} s n(s),
$$

where $n(s)$ is the cluster size distribution. This expression can be evaluated exactly on a Bethe lattice, by using the results of Ref. [14]. Alternatively, using the asymptotic expression for the cluster size distribution [1] one can perform a saddlepoint integration for a general $d$-dimensional system near the percolation threshold (for details, see Ref. [12]). Using the latter method one finds the following relaxation regimes. In the fluid phase, at distance $\epsilon$ from the threshold, one has that the system relaxation is described by a combination of algebraic and stretched exponential decay [15]:

$$
\phi(t, \epsilon) \sim \epsilon^{\beta}\left(\frac{\tau_{\epsilon}}{t}\right)^{c} \exp \left[-\left(\frac{t}{\tau_{\epsilon}}\right)^{y}\right],
$$

where exponents $c$ and $y$ are related to $x, \beta$, and $\gamma$ (the exponent defining the divergence of the mean cluster size at the threshold) by [16]

$$
c=\frac{3 \beta+\gamma}{2(\beta+\gamma)(x+1)}, \quad y=\frac{1}{x+1} .
$$

The relaxation time and the critical decay law turn out to be $\tau_{\epsilon} \sim|\epsilon|^{-\zeta}$ and $\phi(t) \equiv \phi(t, \epsilon=0) \sim t^{-a}$, where

$$
\zeta=x(\beta+\gamma), \quad a=\frac{\beta}{x(\beta+\gamma)} .
$$

In the frozen phase, where ergodicity is broken due to the appearance of a percolating cluster, the asymptotic value of the correlator is $q \sim|\epsilon|^{\beta}$, and the nonarrested part, $\phi(t, \epsilon)-q$, has the same form as Eq. (7) with exponents $c^{\prime}$ and $y^{\prime}$ related to those in the fluid phase by

$$
c^{\prime}=\frac{c / y-1 / 2 d}{1 / y-1 / d}, \quad y^{\prime}=\frac{1-1 / d}{1 / y-1 / d} .
$$

Note that the above exponents depend on the microscopic mechanism responsible for the single cluster relaxation, i.e., on $x$. Nevertheless, one can suitably combine them to get universal scaling laws that only depend on the percolation exponents. We shall come back to this important point later in our comparison with MCT. For the moment, we remark that Eqs. (8) and (9) imply

$$
a \zeta=\beta, \quad \frac{c}{y}=\frac{3 \beta+\gamma}{2(\beta+\gamma)},
$$

and that similar relations can be obviously found between any pair of $a, c, y$, and $\zeta$. Moreover, $y^{\prime}=y, c^{\prime}=c$ in the mean-field limit $d \rightarrow \infty$ [12].

These findings are naturally interpreted in the context of a sol-gel transition where clusters consist of bonded multifunctional monomers and gelation corresponds to the formation of a percolating network of crosslinked polymers [1,17-19]. One can distinguish two cases: (i) If clusters keep their identity for all time and never break, as in chemical (or strong) gels with permanent bonds, then Eq. (7) spans the entire range $t \gtrsim \tau_{\epsilon}$. (ii) When the bonds' lifetime is of the order of the relaxation time, as for physical gels, Eq. (7) is restricted to $t \lesssim \tau_{\epsilon}$, and is eventually followed by a fast exponential decay. This latter case is the one which is more relevant to the present context. We now focus our attention on MCT and show that all these scaling predictions, in their mean-field limit, are met by the $\mathrm{F}_{12}$ schematic model.

Percolation approach versus MCT. In the mean-field percolation theory one has $\beta=\gamma=1$ and the dynamical critical exponents become

$$
c=\frac{1}{x+1}, \quad y=\frac{1}{x+1}, \quad \zeta=2 x, \quad a=\frac{1}{2 x},
$$

from which mean-field universal relations can be derived,

$$
\zeta a=1, \quad \frac{c}{y}=1, \quad c=\frac{2 a}{2 a+1} .
$$


Notice that the first scaling relation in Eq. (13) reproduces the MCT scaling relation Eq. (3). Furthermore, this percolation approach has precise predictions for the MCT solutions, namely, for each value of $\lambda$, the correlator $\phi(t)$ is described, close to the critical point, by an intermediate scaling regime given by Eq. (7), with exponents given by Eqs. (12) and $x$ determined by

$$
\frac{\Gamma^{2}(1-1 / 2 x)}{\Gamma(1-1 / x)}=\lambda
$$

This last relation follows from the third relation of Eq. (3) and the fourth of Eq. (12).

To check the above percolation predictions against the $F_{12}$ schematic model we first consider the simplest case $\lambda=v_{2}=0$ which gives $x=1$ and, from Eq. (12), $y=c=a=1 / 2$ and $\zeta=2$. In this special case, corresponding to $v_{2}=0$ and $\beta=1$, the MCT relaxation dynamics is exactly known [2]:

$$
\Delta \phi(t, \epsilon)=\frac{|\epsilon|}{2}\left[\sqrt{\frac{\tau_{\epsilon}}{\pi t}} \exp \left(-\frac{t}{\tau_{\epsilon}}\right)-\operatorname{erfc}\left(\sqrt{\frac{t}{\tau_{\epsilon}}}\right)\right],
$$

where $\Delta \phi(t, \epsilon)$ is the nonarrested part of the correlator, and $\tau_{\epsilon} \simeq \epsilon^{-2}$ near the transition (with $\epsilon=1-1 / v_{1}$ ). Accordingly, at short times $t / \tau_{\epsilon} \ll 1$ relaxation is algebraic, $\phi(t, \epsilon) \sim t^{-1 / 2}$, while at large times, $t / \tau_{\epsilon} \gg 1$, it is exponentially fast. Expanding Eq. (15) for small $t / \tau_{\epsilon}$ one finds

$$
\Delta \phi(t, \epsilon) \simeq \frac{|\epsilon|}{2} \sqrt{\frac{\tau_{\epsilon}}{\pi t}}\left[1-\sqrt{\frac{\pi t}{\tau_{\epsilon}}}+\frac{t}{\tau_{\epsilon}}\right],
$$

that is, to the leading order in $\sqrt{t / \tau_{\epsilon}}$, Eq. (7) with a normalized relaxation time $\tilde{\tau}_{\epsilon}=\pi \tau_{\epsilon}$. Thus the early and late stage relaxation behaviors are bridged by a scaling regime described by Eq. (7) with exponents exactly matched by the percolation predictions. Figure 1 shows how these three relaxation regimes compare with the exact solution of the $F_{12}$ schematic model (for $\lambda=v_{2}=0$ ).

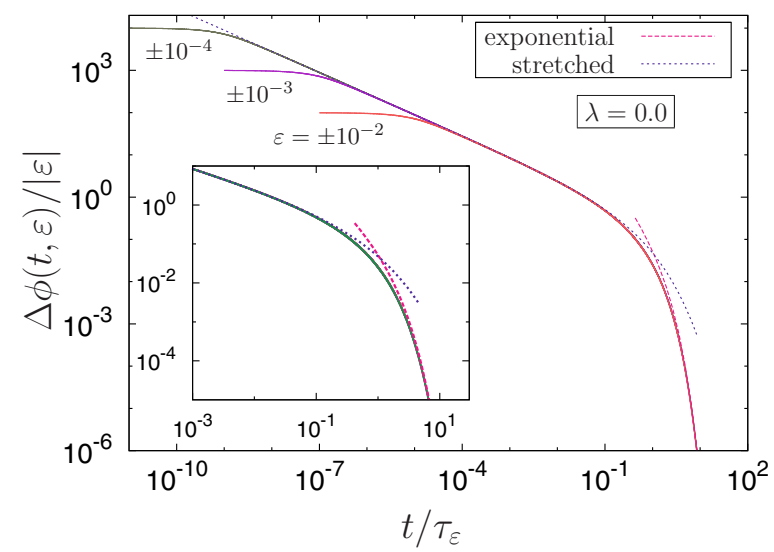

FIG. 1. (Color online) Nonarrested part of correlator $\Delta \phi(t, \epsilon)$ vs rescaled time $t / \tau_{\epsilon}$ for $\lambda=0$. Solid lines are the numerical solution of the MCT schematic model, Eqs. (1) and (2), above $(\epsilon>0)$ and below $(\epsilon<0)$ the transition line. The dotted line is the stretched relaxation regime Eq. (7) obtained by expanding the MCT exact solution to the leading order in $t / \tau_{\epsilon}$. The dashed line represents the late stage exponential decay.
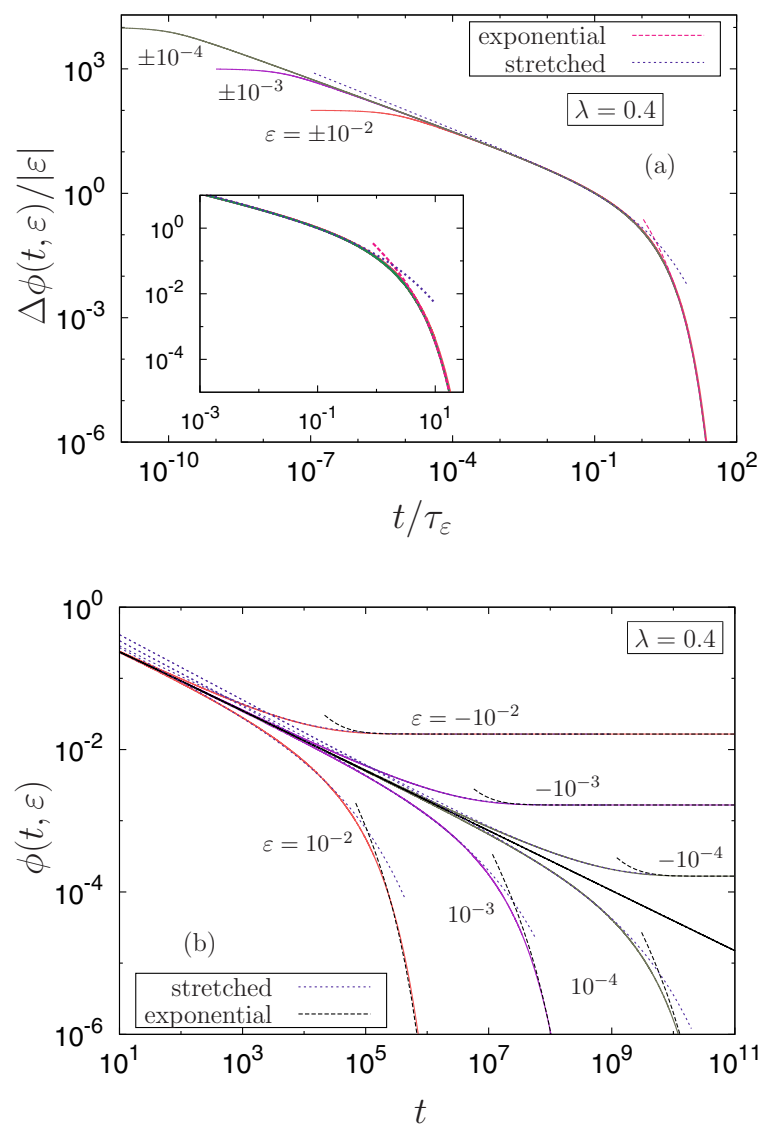

FIG. 2. (Color online) Nonarrested part of correlator $\Delta \phi(t, \epsilon)$ in scaling form (top) and in natural units (bottom) for $\lambda=0.4$. Solid lines are the exact solution of MCT schematic model, Eqs. (1) and (2). The dotted line is the stretched relaxation regime Eq. (7) with exponents determined according to percolation predictions Eqs. (12)-(14) as $x=1.186, c=y=0.457, z=2.372$, and $a=$ 0.422 . The dashed line represents the late stage exponential decay.

For $\lambda>0$ the correlator cannot be expressed in a closed form. Therefore, we have numerically solved MCT Eq. (2) in the continuous glass transition range and found excellent agreement in an extended region of $\lambda$ values. In Fig. 2(a) we show the correlator scaling for $\lambda=0.4$ and, to better appreciate the quality of comparison, we replot in Fig. 2(b) the same set of data in natural units. The characteristic relaxation time $\tau_{\epsilon}$ is defined here as

$$
\tau_{\epsilon}=\int_{0}^{\infty} t \phi(t, \epsilon) d t / \int_{0}^{\infty} \phi(t, \epsilon) d t
$$

and consistently reproduces the expected MCT scaling $\tau_{\epsilon} \sim|\epsilon|^{-\zeta}$.

The natural limits of the present description are reached when $\lambda=v_{2} \rightarrow 1$. In this case the quadratic term of the MCT kernel becomes increasingly important as compared to the linear one and, correspondingly, the intermediate scaling regime shrinks. This is simply understood by considering that the point $\lambda=1$ marks a crossover to a completely distinct critical behavior which is characterized by a discontinuous ergodicity breaking. 


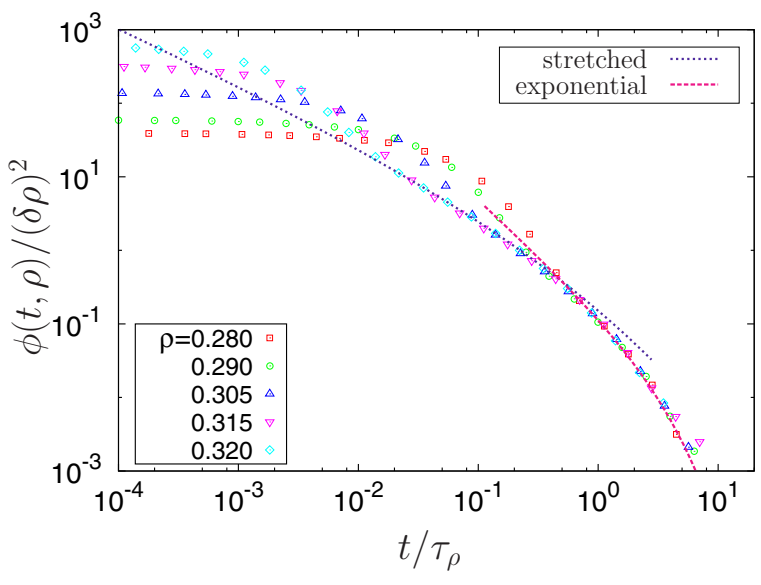

FIG. 3. (Color online) Rescaled persistence function $\phi(t, \rho)$ for a facilitated spin model on a Bethe lattice with order parameter critical exponent $\beta=2$ and critical density $\rho_{\mathrm{c}}=1 / 3$. Relaxation time $\tau_{\rho}$ is computed according to Eq. (17) and $\delta \rho=1-\rho / \rho_{\mathrm{c}}$. The dotted line is the stretched relaxation regime Eq. (7) with exponents determined from the measure of critical relaxation exponent $a$ and Eqs. (8) and (9). The dashed line represents the late stage exponential decay.

Percolation approach versus cooperative facilitation. To substantiate more generally the predictions of the percolation approach to glassy systems with continuously broken ergodicity characterized by $\beta \neq 1$, we finally investigate an instance of cooperative facilitated dynamics on a Bethe lattice [20]. A set of $N$ noninteracting binary spins in a magnetic field favoring up states evolves with a Metropolis-like dynamics in which a randomly chosen spin is flipped if and only if at least $f$ of its $z$ neighbors are down. For $f=3$ and $z=4$ this facilitated dynamics undergoes a continuous ergodicity breaking at a critical density of up spins $\rho_{\mathrm{c}}=1 / 3$. Since the incipient cluster of frozen spins has a fractal structure with no dangling ends, the order parameter critical exponent is $\beta=2$. We have simulated the dynamical behavior of this model with a continuous time algorithm and studied the persistence function $\phi(t)$ (the probability that a spin has never flipped between times 0 and $t$ ) and the relaxation time $\tau_{\rho}$. To compare persistence data with percolation predictions we first measure $a \simeq 0.82$ from the critical decay at $\rho_{\mathrm{c}}$. The remaining critical exponents are then inferred from Eqs. (8) and (9). Note that for this backbone percolation problem the relevant value of the mean-field critical exponent $\gamma$ is $\gamma=d \nu-2 \beta=-1$. By doing so we get $x=\zeta=2 / a \simeq 2.4, y \simeq 0.29$, and $c \simeq 0.73$. Figure 3 shows the rescaled persistence versus $t / \tau_{\rho}$ for several values of the density of up spins $\rho$ near the threshold. We clearly see that also for this facilitated dynamics, the scaling relations Eqs. (4), (5), and (7) provide an excellent description of relaxation behavior.

Conclusions. To summarize, we have established a close analogy between a dynamical percolation approach and glassy systems with a continuous glass transition which is built upon MCT as a mean-field starting point. Consequently, MCT for a type-A transition provides a useful mean-field approach to gelling systems, in the same way as MCT for a type-B transition provides a mean-field framework for structural glasses.

Our percolation approach yields detailed predictions for the critical exponents in any spatial dimension and an intermediate scaling regime of the correlation function. Any finite-dimensional generalization of MCT for systems with continuous transitions should be compared with the above scaling laws, involving universal critical exponents of equilibrium percolation along with a single parameter, governing the local relaxation dynamics of finite clusters. Our framework directly implies an upper critical dimension of 6 and a percolation critical length for such systems.

Some of these quantitative predictions have been previously confirmed by quasiscattering experiments [21] and large scale numerical simulations [12,22] of permanent gels, and we expect they have a much wider relevance for glassy relaxation with continuous ergodicity breaking, including quenched-annealed mixtures [3-5], colloidal gelation [23], and vulcanization [24]. It would be also interesting to revisit in this perspective systems for which a proper definition of a cluster is rather tricky or lacking, such as random-field Ising models and spin glasses [25]. In those cases, $x$ could be inferred indirectly through a measurement of global critical relaxation and thus dynamic scaling laws should be readily tested.

Finally, it would be valuable to generalize the present approach to glassy systems with a two-step relaxation scenario and discontinuous ergodicity breaking. Cooperative facilitation dynamics governed by a bootstrap percolation process suggests that this may be possible [26].

J.J.A. acknowledges partial support from the Brazilian agencies CNPq, Fapergs, CAPES, and from the INCT Sistemas Complexos.
[1] D. Stauffer and A. Aharony, Introduction to Percolation Theory (Taylor \& Francis, London, 1992); M. Sahimi, Applications of Percolation Theory (Taylor \& Francis, London, 1994).

[2] W. Götze, Complex Dynamics of Glass-Forming Liquids (Oxford University Press, Oxford, UK, 2009).

[3] V. Krakoviack, Phys. Rev. Lett. 94, 065703 (2005); Phys. Rev. E 75, 031503 (2007).

[4] J. Kurzidim, D. Coslovich, and G. Kahl, Phys. Rev. Lett. 103, 138303 (2009); K. Kim, K. Miyazaki, and S. Saito, Europhys. Lett. 88, 36002 (2009).
[5] S. Lang, R. Schilling, V. Krakoviack, and T. Franosch, Phys. Rev. E 86, 021502 (2012).

[6] W. Götze, E. Leutheusser, and S. Yip, Phys. Rev. A 23, 2634 (1981); 25, 533 (1982).

[7] G. Biroli, J.-P. Bouchaud, K. Miyazaki, and D. R. Reichman, Phys. Rev. Lett. 97, 195701 (2006).

[8] T. Sarlat, A. Billoire, G. Biroli, and J.-P. Bouchaud, J. Stat. Mech. (2009) P08014.

[9] G. Szamel and E. Flenner, Phys. Rev. Lett. 107, 105505 (2011).

[10] S. Franz, H. Jacquin, G. Parisi, P. Urbani, and F. Zamponi, Proc. Natl. Acad. Sci. USA 109, 18725 (2012). 
[11] S. Franz, G. Parisi, and F. Ricci-Tersenghi, J. Stat. Mech. (2013) L02001.

[12] A. Fierro, T. Abete, and A. Coniglio, J. Chem. Phys. 131, 194906 (2009).

[13] M. Doi and S. F. Edwards, The Theory of Polymer Dynamics (Clarendon, Oxford, UK, 1986).

[14] M. E. Fisher and J. W. Essam, J. Math. Phys. 2, 609 (1961).

[15] Intriguingly, a similar decay appears in the strong coupling regime of the Kardar-Parisi-Zhang equation; see F. Colaiori and M. A. Moore, Phys. Rev. E 63, 057103 (2001).

[16] The value of $x$ must be limited to $x>\beta /(\beta+\gamma)$ otherwise the relations with the exponents do not hold.

[17] P. G. de Gennes, Scaling Concepts in Polymer Physics (Cornell University Press, Ithaca, NY, 1993).

[18] P. J. Flory, The Physics of Polymer Chemistry (Cornell University Press, Ithaca, NY, 1954).

[19] D. Stauffer, A. Coniglio, and M. Adam, Adv. Polym. Sci. 44, 103 (1982).
[20] M. Sellitto, D. De Martino, F. Caccioli, and J. J. Arenzon, Phys. Rev. Lett. 105, 265704 (2010); M. Sellitto, G. Biroli, and C. Toninelli, Europhys. Lett. 69, 496 (2005).

[21] J. E. Martin, J. P. Wilcoxon, and J. Odinek, Phys. Rev. A 43, 858 (1991).

[22] E. Del Gado, A. Fierro, L. de Arcangelis, and A. Coniglio, Phys. Rev. E 69, 051103 (2004); E. Del Gado, L. de Arcangelis, and A. Coniglio, Eur. Phys. J. E 2, 359 (2000).

[23] E. Zaccarelli, J. Phys.: Condens. Matter 19, 323101 (2007); F. Sciortino and P. Tartaglia, Adv. Phys. 54, 471 (2005).

[24] P. M. Goldbart, H. E. Castillo, and A. Zippelius, Adv. Phys. 45, 393 (1996); W. Peng, P. M. Goldbart, and A. J. McKane, Phys. Rev. E 64, 031105 (2001).

[25] A. T. Ogielski, Phys. Rev. B 32, 7384 (1985).

[26] M. Sellitto, Phys. Rev. E 86, 030502(R) (2012); J. J. Arenzon and M. Sellitto, J. Chem. Phys. 137, 084501 (2012). 